PROCEEDINGS OF THE

AMERICAN MATHEMATICAL SOCIETY

Volume 132, Number 7 , Pages 2005-2012

S 0002-9939(03)07325-8

Article electronically published on December 31, 2003

\title{
COMMUTANTS OF REFLEXIVE ALGEBRAS AND CLASSIFICATION OF COMPLETELY DISTRIBUTIVE SUBSPACE LATTICES
}

\author{
PENGTONG LI, SHIJIE LU, AND JIPU MA
}

(Communicated by David R. Larson)

\begin{abstract}
Let $\mathcal{L}$ be a subspace lattice on a normed space $X$ containing a nontrivial comparable element. If $T$ commutes with all the operators in $\operatorname{Alg} \mathcal{L}$, then there exists a scalar $\lambda$ such that $(T-\lambda I)^{2}=0$. Furthermore, we classify the class of completely distributive subspace lattices into subclasses called Type $I^{(n)}$, Type $I I^{(n)}$ and Type $I I I$, respectively. It is shown that nontrivial nests or, more generally, completely distributive subspace lattices with a comparable element are Type $I^{(1)}$, and that nontrivial atomic Boolean subspace lattices are Type $I I^{(0)}$.
\end{abstract}

\section{INTRODUCTION AND PRELIMINARIES}

Throughout this paper, unless otherwise indicated, we denote by $X$ a fixed normed space over the complex number field $\mathbb{C}$, and by $X^{*}$ the dual of $X$. The terms "subspace" and "operator" will be used for "closed subspace of $X$ " and "bounded linear operator acting on $X$ ", respectively. The algebra of all operators is denoted by $\mathcal{B}(X)$. For a subset $\mathcal{A}$ of $\mathcal{B}(X)$, a subspace $L$ of $X$ and an operator $T \in \mathcal{B}(X)$, we will employ the following notation. We use $\mathcal{A}^{\prime}$ for the commutant of $\mathcal{A}$, which is the set of all operators commuting with every operator in $\mathcal{A}$, and $\mathcal{A}^{\prime \prime}$ for the double commutant. Denote by $T^{*}$ the adjoint of $T$, by $\left.T\right|_{L}$ the restriction of $T$ to $L$ and by $I_{L}$ the identity operator on $L$. Also, $L^{\perp}$ denotes the annihilator of $L$, which is the set $\left\{f \in X^{*}: f(x)=0\right.$ for all $\left.x \in L\right\}$. The notation $[\mathcal{A L}]$ stands for the subspace generated by the set $\{A x: A \in \mathcal{A}, x \in L\}$. For $x \in X$ and $f \in X^{*}$, the operator $x \otimes f$ is defined by $y \mapsto f(y) x$. This operator has rank one if and only if both $x$ and $f$ are nonzero. For a family $\mathcal{L}$ of subspaces, we say that $\mathcal{L}$ is a subspace lattice if it contains $(0)$ and $X$, and is closed under the operations $\vee$ (closed linear span) and $\cap$ (set-theoretic intersection). A totally ordered subspace lattice is called a nest. A subspace lattice $\mathcal{L}$ is called complemented if for every $L \in \mathcal{L}$ there is an element $L^{\prime} \in \mathcal{L}$ such that $L \vee L^{\prime}=X$ and $L \cap L^{\prime}=(0)$, and distributive if the identity $L \cap(M \vee N)=(L \cap M) \vee(L \cap N)$ and its dual hold for all $L, M, N \in \mathcal{L}$. A complemented and distributive subspace lattice is called a Boolean subspace lattice. An element $L$ in a subspace lattice $\mathcal{L}$ is called an atom if, whenever $K \in \mathcal{L}$ such

Received by the editors November 19, 2001 and, in revised form, March 16, 2003.

2000 Mathematics Subject Classification. Primary 47L35, 47L75.

Key words and phrases. Reflexive algebras, commutants, complete distributivity, comparable elements, rank one operators. 
that $(0) \subseteq K \subseteq L$, then either $K=(0)$ or $K=L$; moreover, $\mathcal{L}$ is called atomic if each element of $\mathcal{L}$ is the closed linear span of the atoms it contains. For standard definitions concerning completely distributive subspace lattices see [5], [10]. From 12] we know that a subspace lattice is completely distributive if and only if it is strongly reflexive.

Proposition 1.1 ([9], [12]). Let $\mathcal{L}$ be a subspace lattice on $X$. Then the following are equivalent:

(i) $\mathcal{L}$ is completely distributive.

(ii) $L=L_{\#}:=\bigvee\left\{K \in \mathcal{L}: L \nsubseteq K_{-}\right\}$holds for every $L \in \mathcal{L}$.

(iii) $L=L_{*}:=\bigcap\left\{K_{-}: K \in \mathcal{L}, K \nsubseteq L\right\}$ holds for every $L \in \mathcal{L}$.

Here $K_{-}$is defined as the subspace $\bigvee\{M \in \mathcal{L}: K \nsubseteq M\}$.

An element $L$ in a subspace lattice $\mathcal{L}$ is said to be a comparable element if for each $M \in \mathcal{L}$ either $M \subseteq L$ or $L \subset M$. Hereafter, we always assume that a comparable element $L$ is nontrivial, that is, $L \neq(0), X$.

For any family $\mathcal{L}$ of subspaces, $\operatorname{let} \operatorname{Alg} \mathcal{L}$ denote the algebra of all operators that leave every subspace in $\mathcal{L}$ invariant. Dually, for any family $\mathcal{A}$ of operators, let Lat $\mathcal{A}$ denote the set of all subspaces that are invariant under each operator in $\mathcal{A}$. It is clear that Lat $\mathcal{A}$ is a subspace lattice. We say that a subspace lattice $\mathcal{L}$ is reflexive if $\mathcal{L}=\operatorname{LatAlg} \mathcal{L}$, and that a subalgebra $\mathcal{A}$ of $\mathcal{B}(X)$ is reflexive if $\mathcal{A}=\operatorname{AlgLat} \mathcal{A}$. The class of reflexive subspace lattices includes nests, atomic Boolean subspace lattices, completely distributive subspace lattices and Lat $\mathcal{A}$ defined above.

It is well known that the commutant of any nest algebra is trivial (see [2]); that is, the commutant is the set of scalar multiples of the identity operator $I$. Moreover, Lambrou in 9. proved that if $\mathcal{L}$ is a completely distributive subspace lattice on a normed space $X$ containing a comparable element, then $(\operatorname{Alg} \mathcal{L})^{\prime}$ is trivial. But, when $X$ is a Hilbert space and the complete distributivity of $\mathcal{L}$ is removed, Gilfeather and Larson in 3] obtained that $(\operatorname{Alg} \mathcal{L})^{\prime}$ is still trivial. These results lead naturally to the following question: if $\mathcal{L}$ is a subspace lattice on a normed space $X$ containing a comparable element (the complete distributivity of $\mathcal{L}$ is not assumed), what is $(\operatorname{Alg} \mathcal{L})^{\prime}$ ? Our first purpose is to investigate this question, and we will give a partial answer, that is,

$$
(\operatorname{Alg} \mathcal{L})^{\prime} \subseteq\left\{T \in \mathcal{B}(X):(T-\lambda I)^{2}=0 \text { for some } \lambda \in \mathbb{C}\right\} .
$$

Our second purpose is to give a classification of completely distributive subspace lattices. We will classify the class of such subspace lattices into subclasses, which are called Type $I^{(n)}$, Type $I I^{(n)}$ and Type $I I I$, respectively. We will see that nontrivial nests or, more generally, completely distributive subspace lattices with a comparable element are Type $I^{(1)}$ and nontrivial atomic Boolean subspace lattices are Type $I I^{(0)}$.

\section{Commutants of Reflexive algebras}

We begin with a lemma, which was proved in 12 for Hilbert spaces and is also valid in normed spaces (see [9]).

Lemma 2.1. If $\mathcal{L}$ is a subspace lattice on $X$, then the rank one operator $x \otimes f \in$ $\operatorname{Alg} \mathcal{L}$ if and only if there exists $L \in \mathcal{L}$ such that $x \in L$ and $f \in L_{-}^{\perp}$.

The main result of this section reads as follows. 
Theorem 2.2. Let $\mathcal{L}$ be a subspace lattice on $X$ containing a comparable element. If $T \in(A \lg \mathcal{L})^{\prime}$, then there exists a scalar $\lambda \in \mathbb{C}$ such that

(i) $\left.T\right|_{X_{\#}}=\lambda I_{X_{\#}}$ and $\left.T^{*}\right|_{\left(X^{*}\right)_{\mathcal{L}}}=\lambda I_{\left(X^{*}\right)_{\mathcal{L}}}$. Here $X_{\#}$ is as in Proposition 1.1, and $\left(X^{*}\right)_{\mathcal{L}}$ denotes the weak $k^{*}$ closed subspace of $X^{*}$ generated by $\bigcup\left\{M_{-}^{\perp}: M \in\right.$ $\mathcal{L}, M \neq(0)\}$

(ii) $(T-\lambda I)^{2}=0$.

Proof. Let $T \in(\operatorname{Alg} \mathcal{L})^{\prime}$. Assuming that $M \in \mathcal{L}$ with $M \neq(0)$ and $M_{-} \neq X$, we claim that there exists $\lambda_{M} \in \mathbb{C}$ such that $\left.T\right|_{M}=\lambda_{M} I_{M}$ and $\left.T^{*}\right|_{M_{\perp}}=\lambda_{M} I_{M_{-}}$. To see this, let $x_{1}, x_{2} \in M$ and $f_{1}, f_{2} \in M_{-}^{\perp}$ be arbitrary and nonzero. By Lemma 2.1 we have $x_{1} \otimes f_{1}, x_{1} \otimes f_{2}, x_{2} \otimes f_{1} \in \operatorname{Alg} \mathcal{L}$, and hence

$$
\begin{aligned}
& T\left(x_{1} \otimes f_{1}\right)=\left(x_{1} \otimes f_{1}\right) T, \\
& T\left(x_{1} \otimes f_{2}\right)=\left(x_{1} \otimes f_{2}\right) T, \\
& T\left(x_{2} \otimes f_{1}\right)=\left(x_{2} \otimes f_{1}\right) T .
\end{aligned}
$$

We then have from $(2.1)$ that $\left(T x_{1}\right) \otimes f_{1}=x_{1} \otimes\left(T^{*} f_{1}\right)$, which implies that there exists $\lambda_{M} \in \mathbb{C}$ depending on $M$ such that

$$
T x_{1}=\lambda_{M} x_{1}, T^{*} f_{1}=\lambda_{M} f_{1} .
$$

In a similar way, it follows from (2.2) and (2.3) that there exist two scalars $\lambda_{1}, \lambda_{2} \in$ $\mathbb{C}$ such that

$$
\begin{aligned}
& T x_{1}=\lambda_{1} x_{1}, T^{*} f_{2}=\lambda_{1} f_{2}, \\
& T x_{2}=\lambda_{2} x_{2}, T^{*} f_{1}=\lambda_{2} f_{1} .
\end{aligned}
$$

Thus from (2.4), (2.5) and (2.6) we can see that $\lambda_{M}=\lambda_{1}=\lambda_{2}$. Since $x_{1}, x_{2}, f_{1}$ and $f_{2}$ are arbitrary, $T x=\lambda_{M} x$ for every $x \in M$ and $T^{*} f=\lambda_{M} f$ for every $f \in M_{-}^{\perp}$. This proves the claim.

Let $L \in \mathcal{L}$ be a comparable element. Then

$$
L_{-}=\bigvee\{K \in \mathcal{L}: L \nsubseteq K\}=\bigvee\{K \in \mathcal{L}: K \subset L\} \subseteq L .
$$

Since $L \neq X$, we have $L_{-} \neq X$. It follows from the above claim that there exists $\lambda \in \mathbb{C}$ such that

$$
\left.T\right|_{L}=\lambda I_{L} \text { and }\left.T^{*}\right|_{L_{\perp}^{\perp}}=\lambda I_{L_{\perp}^{\perp}}
$$

For any $M \in \mathcal{L}$ with $M \neq(0)$ and $M_{-} \neq X$, applying the above claim again, there exists $\lambda_{M} \in \mathbb{C}$ such that $\left.T\right|_{M}=\lambda_{M} I_{M}$ and $\left.T^{*}\right|_{M_{-}^{\perp}}=\lambda_{M} I_{M_{-}^{\perp}}$. Since $L$ and $M$ are nonzero and $L$ is comparable, the subspace $L \cap M$ is not zero. Then it is easy to see that $\lambda_{M}=\lambda$. Recalling that $X_{\#}=\bigvee\left\{M \in \mathcal{L}: M_{-} \neq X\right\}$ and the definition of $\left(X^{*}\right)_{\mathcal{L}}$, we have $\left.T\right|_{X_{\#}}=\lambda I_{X_{\#}}$ and $\left.T^{*}\right|_{\left(X^{*}\right)_{\mathcal{L}}}=\lambda I_{\left(X^{*}\right)_{\mathcal{L}}}$. This establishes the conclusion (i).

Next we will prove the conclusion (ii). By (2.7) we have $L^{\perp} \subseteq L_{-}^{\perp}$. Consequently, $\left.T^{*}\right|_{L^{\perp}}=\lambda I_{L^{\perp}}$ by (2.8). Let $x \in X$ be arbitrary. For any $f \in L^{\perp}$, one has

$$
f(T x-\lambda x)=f(T x)-\lambda f(x)=\left(T^{*} f\right)(x)-\lambda f(x)=(\lambda f)(x)-\lambda f(x)=0 .
$$

Therefore, $T x-\lambda x \in L$. By (2.8) again, $T(T x-\lambda x)=\lambda(T x-\lambda x)$, which implies that $(T-\lambda I)^{2} x=0$. This means that $(T-\lambda I)^{2}=0$. The proof is complete. 
Corollary 2.3. Let $\mathcal{L}$ be a subspace lattice on $X$ containing a comparable element. If either $X=X_{\#}$ or $(0)=(0)_{*}$, where $X_{\#}$ and $(0)_{*}$ are as in Proposition 1.1, then $(\mathrm{Alg} \mathcal{L})^{\prime}$ is trivial.

Proof. For the case that $(0)=(0)_{*}$, it is easily seen that $X^{*}=\left(X^{*}\right)_{\mathcal{L}}$, where $\left(X^{*}\right)_{\mathcal{L}}$ is as in Theorem 2.2. Thus the desired result follows immediately from Theorem 2.2 .

By Proposition 1.1, the condition that $\mathcal{L}$ satisfies either $X=X_{\#}$ or $(0)=(0)_{*}$ is much weaker than the condition that $\mathcal{L}$ is completely distributive. So Corollary 2.3 strengthens the result of Lambrou mentioned in Section 1.

Corollary 2.4. Let $\mathcal{L}$ be a subspace lattice on $X$ satisfying either $X{ }_{-} \neq X$ or $(0)_{+} \neq(0)$, where $(0)_{+}=\bigcap\{M \in \mathcal{L}: M \neq(0)\}$. Then $(\operatorname{Alg} \mathcal{L})^{\prime}$ is trivial.

Proof. We may suppose that $\mathcal{L} \neq\{(0), X\}$. If $X_{-} \neq X$, then $X_{\#}=X$ and $X_{-}$is a comparable element. If $(0)_{+} \neq(0)$, then $(0)_{*} \subseteq\left((0)_{+}\right)_{-}=(0)$. So $(0)_{*}=(0)$ and $(0)_{+}$is a comparable element. Hence the result holds true by Corollary 2.3 .

\section{Classification of COMPletely Distributive Subspace lattices}

In this section, we will give a classification of completely distributive subspace lattices. Let us first characterize the rank one operators in the Jacobson radical of reflexive algebras. Recall that the Jacobson radical of an arbitrary algebra is defined to be the intersection of the kernels of all strictly transitive representations of the algebra. But, it is well known that if $\mathcal{A}$ is a Banach algebra with an identity, then the Jacobson radical of $\mathcal{A}$, denoted by $\operatorname{Rad}(\mathcal{A})$, can be reformed as follows (see [1]):

$$
\begin{aligned}
\operatorname{Rad}(\mathcal{A}) & =\{T \in \mathcal{A}: T A \text { is quasinilpotent for all } A \in \mathcal{A}\} \\
& =\{T \in \mathcal{A}: A T \text { is quasinilpotent for all } A \in \mathcal{A}\} .
\end{aligned}
$$

It is worthy to note that $\operatorname{Rad}(\mathcal{A})$ is a two-sided ideal of $\mathcal{A}$.

The next lemma is a slight variant of Lemma 3 and the note after that of [6].

Lemma 3.1. Let $\mathcal{L}$ be a reflexive subspace lattice on $X$, and let the rank one operator $x \otimes f \in \operatorname{Alg} \mathcal{L}$. Then $x \otimes f \in \operatorname{Rad}(\operatorname{Alg} \mathcal{L})$ if and only if there exists a subspace $L \in \mathcal{L}$ such that $x \in L$ and $f \in L^{\perp}$.

Proof. Suppose that $x \otimes f \in \operatorname{Rad}(\operatorname{Alg} \mathcal{L})$. Putting $L=[(\operatorname{Alg} \mathcal{L}) x]$, then $L \in$ Lat $\operatorname{Alg} \mathcal{L}=\mathcal{L}$ since $\mathcal{L}$ is reflexive. In fact, $L$ is the least subspace in $\mathcal{L}$ containing $x$. It remains to show that $f \in L^{\perp}$. For this purpose, it suffices to prove that $f(A x)=0$ for each $A \in \operatorname{Alg} \mathcal{L}$. Assume on the contrary that there is an $A \in \operatorname{Alg} \mathcal{L}$ with $f(A x) \neq 0$. Without loss of generality, let $f(A x)>0$. Then

$$
\lim _{n}\left\|(A x \otimes f)^{n}\right\|^{\frac{1}{n}}=\lim _{n}|f(A x)|^{\frac{n-1}{n}}\|A x \otimes f\|^{\frac{1}{n}}=|f(A x)| \neq 0 .
$$

Hence $A x \otimes f$ is not quasinilpotent, contradicting that $x \otimes f \in \operatorname{Rad}(\operatorname{Alg} \mathcal{L})$.

Conversely, suppose that there exists $L \in \mathcal{L}$ satisfying $x \in L$ and $f \in L^{\perp}$. For any $A \in \operatorname{Alg} \mathcal{L}$, then $A x \in L$ and so $f(A x)=0$, which implies that $A x \otimes f$ is quasinilpotent. Therefore, $x \otimes f \in \operatorname{Rad}(\operatorname{Alg} \mathcal{L})$. 
Corollary 3.2. Let $\mathcal{N}$ be a nest on $X$. Then the rank one operator $x \otimes f \in$ $\operatorname{Rad}(\operatorname{Alg} \mathcal{N})$ if and only if there exists $L \in \mathcal{N}$ such that $x \in L$ and $f \in L^{\perp}$.

Proof. Since $\mathcal{N}$ is a nest, we have $L_{-} \subseteq L$ for all $L \in \mathcal{N}$. If $x \in L$ and $f \in L^{\perp}$ for some $L \in \mathcal{N}$, then $x \otimes f$ must belong to $\operatorname{Alg} \mathcal{N}$ by Lemma 2.1. Thus the desired result holds immediately by Lemma 3.1 .

The above corollary was proved in [4] by different methods when $X$ is a Hilbert space.

In what follows, for a subspace $L$ of $X$, denote

$$
L \otimes L^{\perp}=\left\{x \otimes f: x \in L, f \in L^{\perp}\right\} .
$$

With this notation, we first characterize when an element in a subspace lattice is comparable.

Proposition 3.3. Let $\mathcal{L}$ be a subspace lattice on $X$ and $L \in \mathcal{L}$. Then $L$ is a comparable element if and only if $L \otimes L^{\perp} \subseteq \operatorname{Alg} \mathcal{L}$.

Proof. Suppose first that $L$ is comparable. By (2.7) we have $L_{-} \subseteq L$. It follows from Lemma 2.1 that $L \otimes L^{\perp} \subseteq \operatorname{Alg} \mathcal{L}$.

Conversely, assume that $L \otimes L^{\perp} \subseteq \operatorname{Alg} \mathcal{L}$. Let $M \in \mathcal{L}$ be arbitrary. If $M \nsubseteq L$, then there exist $y \in M$ and $f \in L^{\perp}$ such that $f(y) \neq 0$. Thus, for any $x \in L$, we have

$$
x=\frac{1}{f(y)}(x \otimes f) y \in\left[\left(L \otimes L^{\perp}\right) M\right] \subseteq[(\operatorname{Alg} \mathcal{L}) M] \subseteq M,
$$

and so $L \subset M$. Therefore, $L$ is a comparable element.

Theorem 3.4. Let $\mathcal{L}$ be a completely distributive subspace lattice on $X$. Then the following are equivalent:

(i) $\mathcal{L}$ is an atomic Boolean subspace lattice;

(ii) $L \otimes L^{\perp} \cap \operatorname{Alg} \mathcal{L}=\{0\}$ for every $L \in \mathcal{L}$;

(iii) $\operatorname{Alg} \mathcal{L}$ is semisimple, that is, $\operatorname{Rad}(\operatorname{Alg} \mathcal{L})=\{0\}$.

Proof. The equivalence of (i) and (iii) is shown in [7] for Hilbert spaces, but the proof can be adapted in a trivial way to Banach spaces. Also, (iii) implies (ii) by Lemma 3.1.

Suppose that (ii) holds. Letting $T \in \operatorname{Rad}(\operatorname{Alg} \mathcal{L})$, we want to show that $T=0$. Assume on the contrary that $T \neq 0$. Since $X=\bigvee\left\{L \in \mathcal{L}: L_{-} \neq X\right\}$, there exists $L \in \mathcal{L}$ with $L_{-} \neq X$ such that $T x \neq 0$ for some $x \in L$. For any $f \in L_{-}^{\perp}$, $x \otimes f \in \operatorname{Alg} \mathcal{L}$ by Lemma 2.1, and hence $T x \otimes f$ is quasinilpotent. Thus $f(T x)=0$, and consequently $T x \in L_{-}$. Picking a nonzero $g \in L_{-}^{\perp}$, then $0 \neq T x \otimes g \in$ $L_{-} \otimes L_{-}^{\perp} \cap \operatorname{Alg} \mathcal{L}$. This is a contradiction. Therefore (iii) holds, and the proof is complete.

The next lemma is one of the main results from [9].

Lemma 3.5. If $\mathcal{L}$ is a completely distributive subspace lattice on $X$, then there exists a unique atomic Boolean sublattice $\mathcal{L}_{a}$ of $\mathcal{L}$ such that $(\operatorname{Alg} \mathcal{L})^{\prime \prime}=\operatorname{Alg} \mathcal{L}_{a}$.

For the purpose of this section, we now introduce some notation. Let $\mathcal{L}$ be a completely distributive subspace lattice on $X$. First, denote by $\mathcal{L}_{1}$ the least subspace lattice containing the set

$$
\left\{L \in \mathcal{L}: L \otimes L^{\perp} \cap \operatorname{Alg} \mathcal{L}=\{0\}\right\}
$$


that is, $\mathcal{L}_{1}$ is the subspace lattice generated by the above set. Second, denote by $\mathcal{L}_{2}$ the least subspace lattice containing the set

$$
\left\{L \in \mathcal{L}_{1}: L \otimes L^{\perp} \cap \operatorname{Alg} \mathcal{L}_{1}=\{0\}\right\} .
$$

Continuing the above procedure in an obvious way, we obtain a sequence $\left\{\mathcal{L}_{n}\right\}_{n=1}^{\infty}$ of subspace lattices with the property that for any positive integer $n, \mathcal{L}_{n+1}$ is the least subspace lattice containing the set

$$
\left\{L \in \mathcal{L}_{n}: L \otimes L^{\perp} \cap \operatorname{Alg} \mathcal{L}_{n}=\{0\}\right\} .
$$

It is clear that a subspace sublattice of a completely distributive subspace lattice is still completely distributive. Thus, all $\mathcal{L}_{n}$ defined above are completely distributive. Moreover, we have

Theorem 3.6. Let $\mathcal{L}$ be a completely distributive subspace lattice on $X$ and let $\mathcal{L}_{0}$ denote $\mathcal{L}$. Then the following hold true:

(i) $\mathcal{L}_{0} \supseteq \mathcal{L}_{1} \supseteq \cdots \supseteq \mathcal{L}_{n} \supseteq \mathcal{L}_{n+1} \supseteq \cdots \supseteq \mathcal{L}_{a}$, where $\mathcal{L}_{a}$ is as in Lemma 3.5. Consequently, $\operatorname{Alg} \mathcal{L}_{0} \subseteq \operatorname{Alg} \mathcal{L}_{1} \subseteq \cdots \subseteq \operatorname{Alg} \mathcal{L}_{n} \subseteq \operatorname{Alg} \mathcal{L}_{n+1} \subseteq \cdots \subseteq \operatorname{Alg} \mathcal{L}_{a}$.

(ii) If there is a natural number $n$ such that $\mathcal{L}_{n}=\mathcal{L}_{n+1}$, then $\mathcal{L}_{m}=\mathcal{L}_{n}$ holds for all $m>n$.

(iii) If there is a natural number $n$ such that $\mathcal{L}_{n}$ contains a comparable element, then $\mathcal{L}_{n+1}=\{(0), X\}$.

(iv) If there is a natural number $n$ such that $\mathcal{L}_{n}$ is an atomic Boolean subspace lattice, then $\mathcal{L}_{n}=\mathcal{L}_{n+1}$.

Proof. (i) For any natural number $n$, obviously $\mathcal{L}_{n} \supseteq \mathcal{L}_{n+1}$; it remains to prove that $\mathcal{L}_{n} \supseteq \mathcal{L}_{a}$. We show this by induction. It is clear that $\mathcal{L}_{0} \supseteq \mathcal{L}_{a}$. Suppose $\mathcal{L}_{n} \supseteq \mathcal{L}_{a}$. Let $L \in \mathcal{L}_{a}$ be arbitrary. Then $L \otimes L^{\perp} \cap \operatorname{Alg} \mathcal{L}_{n} \subseteq L \otimes L^{\perp} \cap \operatorname{Alg} \mathcal{L}_{a}$. But, since $\mathcal{L}_{a}$ is an atomic Boolean subspace lattice, it follows from Theorem 3.4 that $L \otimes L^{\perp} \cap \operatorname{Alg} \mathcal{L}_{a}=\{0\}$. So we must have $L \otimes L^{\perp} \cap \operatorname{Alg} \mathcal{L}_{n}=\{0\}$, which implies that $L \in \mathcal{L}_{n+1}$. Therefore, $\mathcal{L}_{n+1} \supseteq \mathcal{L}_{a}$, and (i) holds.

(ii) Immediate by definitions.

(iii) Suppose $\mathcal{L}_{n}$ contains a comparable element $L_{0}$. Let $L \in \mathcal{L}_{n}$ such that $L \otimes L^{\perp} \cap \operatorname{Alg} \mathcal{L}_{n}=\{0\}$. Then either $L \supseteq L_{0}$ or $L \subset L_{0}$. If $L \supseteq L_{0}$, let $f \in L^{\perp}$ be arbitrary. Noting that $L_{0} \neq(0)$, choose a nonzero $x \in L_{0}$; then $x \otimes f \in L \otimes L^{\perp}$. On the other hand, $x \otimes f \in L_{0} \otimes L_{0}^{\perp} \subseteq \operatorname{Alg} \mathcal{L}_{n}$ by Proposition 3.3. Hence $x \otimes f=0$ by the assumption on $L$, and then $f=0$ since $x \neq 0$. This shows that $L=X$. In the case that $L \subset L_{0}$, we can similarly get $L=(0)$. Thus $\mathcal{L}_{n+1}=\{(0), X\}$.

(iv) Immediate by Theorem 3.4.

The proof is complete.

Now we are in a position to classify completely distributive subspace lattices.

Definition 3.7. Let $\mathcal{L}$ be a completely distributive subspace lattice on $X$, and let $\mathcal{L}_{0}$ denote $\mathcal{L}$.

(i) $\mathcal{L}$ is called Type $I^{(n)}$ if there is a natural number $n$ such that $\mathcal{L}_{n-1} \neq \mathcal{L}_{n}=$ $\{(0), X\}$.

(ii) $\mathcal{L}$ is called Type $I I^{(n)}$ if there is a natural number $n$ such that $\mathcal{L}_{n-1} \neq \mathcal{L}_{n}=$ $\mathcal{L}_{n+1} \neq\{(0), X\}$.

(iii) $\mathcal{L}$ is called Type III if $\mathcal{L}_{n-1} \neq \mathcal{L}_{n}$ for every natural number $n$. (Here we take $\mathcal{L}_{-1} \neq \mathcal{L}_{0}$.) 
Proposition 3.8. Let $\mathcal{L}$ be a completely distributive subspace lattice on $X$.

(i) If $\mathcal{L}$ contains a comparable element, then $\mathcal{L}$ is Type $I^{(1)}$; in particular, nontrivial nests are Type $I^{(1)}$.

(ii) If $\mathcal{L}$ is Type $I^{(n)}$ for a natural number $n$, then $(\operatorname{Alg} \mathcal{L})^{\prime}$ is trivial.

(iii) If $\mathcal{L}$ is a nontrivial atomic Boolean subspace lattice, then $\mathcal{L}$ is Type $I I^{(0)}$.

Proof. (i) Immediate by the conclusion (iii) of Theorem 3.6.

(ii) If $\mathcal{L}$ is Type $I^{(n)}$, then $\mathcal{L}_{n}=\{(0), X\}$. Applying Lemma 3.5 and Theorem 3.6, we have $\operatorname{Alg} \mathcal{L} \subseteq \operatorname{Alg} \mathcal{L}_{n} \subseteq(\operatorname{Alg} \mathcal{L})^{\prime \prime}$. Then $(\operatorname{Alg} \mathcal{L})^{\prime}=\left(\operatorname{Alg} \mathcal{L}_{n}\right)^{\prime}=\{\lambda I: \lambda \in \mathbb{C}\}$.

(iii) Immediate by the conclusion (iv) of Theorem 3.6.

This completes the proof.

Notice that our classification scheme is essentially a lattice scheme, since the nonzero $L$ in $\mathcal{L}_{n}$ are simply those generated from the $L$ in $\mathcal{L}_{n-1}$ with $L \vee L_{-}=X$. On the other hand, the $\mathcal{L}_{a}$ in Lemma 3.5 depend very much on the geometry of $\mathcal{L}$. Indeed, in Chapter 7 of [1, there are conditions that show that (even finite) isomorphic atomic Boolean subspace lattices might have different $\mathcal{L}_{a}$ (see Corollary 7.7 there).

\section{ACKNOWLEDGMENTs}

The authors wish to express their thanks to the referee and Professor W. E. Longstaff for their many valuable suggestions.

\section{REFERENCES}

[1] S. Argyros, M. S. Lambrou and W. E. Longstaff, Atomic Boolean subspace lattices and applications to the theory of bases, Mem. Amer. Math. Soc. 91, no. 445 (1991). MR 92m:46022

[2] K. Davidson, Nest algebras, Pitman Research Notes in Math. Ser. 191, Longman Scientific and Technical, Harlow, 1988. MR 90f:47062

[3] F. Gilfeather and D. R. Larson, Nest-subalgebras of von Neumann algebras: Commutants modulo compacts and distance estimates, J. Operator Theory 7 (1982), 279-302. MR 84g:47040

[4] W. Gong and J. Zhu, Strong-principal bimodules of nest algebras, Proc. Amer. Math. Soc. (2) 115 (1992), 435-440. MR 93b:47088

[5] A. Hopenwasser, Complete distributivity, Proc. Sympos. Pure Math. 51 (1990), 285-305. MR 92a: 47052

[6] A. Katavolos and E. Katsoulis, Semisimplicity in operator algebras and subspace lattices, J. London Math. Soc. (2) 42 (1990), 365-372. MR 92b:47066

[7] M. S. Lambrou, Complete atomic Boolean lattices, J. London Math. Soc. (2) 15 (1977), 387-390. MR 56:2891

[8] M. S. Lambrou, Semisimple completely distributive lattices are Boolean algebras, Proc. Amer. Math. Soc. 68 (1978), 217-219. MR 57:3030

[9] M. S. Lambrou, Approximants, commutants and double commutants in normed algebras, J. London Math. Soc. (2) 25 (1982), 499-512. MR 84f:47053

[10] M. S. Lambrou, Completely distributive lattices, Fundamenta Mathematica 119 (1983), 227240. MR 85g:06008

[11] D. R. Larson, On the structure of certain reflexive operator algebras, J. Funct. Anal. (3) 31 (1979), 275-292. MR 80i:47062 
[12] W. E. Longstaff, Strongly reflexive lattices, J. London Math. Soc. (2) 11 (1975), 491-498. MR 52:15036

Department of Mathematics, College of Science, Nanjing University of Aeronautics and Astronautics, Nanjing 210016, People's Republic of China

E-mail address: pengtonglee@vip.sina.com

Department of Mathematics, Zhejiang University, Hangzhou 310027, People's RepubLIC OF CHINA

Current address: City College, Zhejiang University, Hangzhou 310015, People's Republic of China

E-mail address: lusj@zucc.edu.cn

Department of Mathematics, Nanjing University, Nanjing 210093, People's Republic of China 